\title{
Metabolic Inhibitors Reversibly Alter the Basal Membrane Potential of the Gecko Retinal Pigment Epithelium
}

\author{
EDWIN R. GRIFF \\ Department of Biological Sciences, University of Cincinnati, ML 006, Cincinnati, OH 45221-0006, \\ U.S.A.
}

(Received and accepted 31 July 1989)

\begin{abstract}
The effects of metabolic inhibitors on the apical and basal membrane potentials were studied in the isolated retinal pigment epithelium of the lizard Gekko gekko. Adding dinitrophenol or cyanide or cooling the tissue to $15^{\circ} \mathrm{C}$ first depolarized the apical membrane and then hyperpolarized the basal membrane. The basal hyperpolarization was accompanied by an apparent increase in basal resistance. These effects were fully reversible. Adding ouabain to inhibit specifically the apical $\mathrm{Na}^{+}-\mathrm{K}^{+}$pump irreversibly depolarized the apical membrane but did not produce a basal membrane hyperpolarization. Dinitrophenol. cyanide and azide also reversibly inhibited a basal membrane response that was evoked by changing the apical potassium concentration. Ouabain did not inhibit this potassium-evoked basal response. These results suggest that metabolic inhibitors will be useful tools to study RPE basal membrane function.

Key words : retinal pigment epithelium: RPE; electrophysiology ; dinitrophenol ; ouabain; cyanide : basal membrane; gecko.
\end{abstract}

\section{Introduction}

In common with all epithelia, the retinal pigment epithelium (RPE) has two distinct membranes. An apical membrane faces the photoreceptors of the neural retina and a basal membrane faces the choroidal blood supply. Light-evoked responses in the amphibian RPE (bullfrog) are generated at the apical membrane (e.g. Steinberg and Miller, 1979) whereas RPE responses of reptiles (lizard), birds (chick), and mammals (cat) respond with potentials generated at both the apical and basal membranes (Steinberg, Linsenmeier and Griff, 1985 ; Gallemore and Steinberg, $1989 \mathrm{~b})$. The potentials generated at the basal membrane in the 'mammalian type' RPE contribute to the fast oscillation trough and light peak of the DC-electroretinogram (Marmor and Lurie, 1979; Steinberg et al., 1985; Van Norren and Heynen, 1986).

The retina of the Tokay gecko, Gekko gekko, has provided an in vitro model to study RPE basal membrane mechanisms (Griff and Steinberg, 1982a, b, 1984). For example, the light-evoked decrease in subretinal potassium concentration produces first a hyperpolarization of the apical membrane followed by a delayed hyperpolarization of the basal membrane. The basal hyperpolarization is accompanied by a decrease in basal membrane conductance (Griff and Steinberg, 1984: Linsenmeier and Steinberg, 1984). Using an RPE preparation isolated from the neural retina (isolated RPE), Griff and Steinberg (1984) demonstrated that a delayed basal hyperpolarization could be produced solely by lowering the potassium concentration outside the apical membrane, $\left[\mathrm{K}^{+}\right]_{\mathrm{ap}}$. The mechanisms that generate this basal hyperpolarization and the intracellular steps that link it to the change in subretinal potassium are unknown. In fact. except for the bullfrog, the electrophysiology of the
RPE basal membrane is not well understood (but see Joseph and Miller, in press; Gallemore and Steinberg, 1989 b).

In the present study, the effects of some widely used metabolic inhibitors were examined to investigate further the gecko isolated RPE, and in particular, potentials that originate at basal membrane. Each inhibitor produced a change in the basal membrane potential. While the specific mechanisms by which each inhibitor acts in the RPE were not determined. the results suggest that these inhibitors will be useful tools to study RPE function.

\section{Materials and Methods}

\section{Preparation}

Tokay geckos, Gekko gekko, were obtained from Charles Sullivan, Inc., Nashville, TN, kept in aquaria and fed a diet of crickets. Animals were dark-adapted for $12 \mathrm{hr}$, refrigerated for $2 \mathrm{hr}$ to facilitate handling, and anesthetized by packing in ice. The eyes were enucleated under dim red light, sectioned behind the lens, and the posterior portion of the eye submerged in perfusate. The retina was removed and discarded and the remaining RPE-choroid was dissected free from the sclera and mounted in a chamber as previously described for bullfrog (Miller and Steinberg, 1977; Oakley, 1977). The area of exposed tissue was $0.07 \mathrm{~cm}^{2}$. After enucleation, the animals were killed.

Both sides of the tissue were continuously superfused at $2-3 \mathrm{ml} \mathrm{min}^{-1}$ by a gravity feed system from two large reservoirs in which each solution was bubbled with $95 \% \mathrm{O}_{2}$ and $5 \% \mathrm{CO}_{\mathrm{z}}$. The control perfusate was a modified Ringer's solution having the following composition (mM): $82.5 \mathrm{NaCl}$, $27.5 \mathrm{NaHCO}_{3} . \quad 2.0 \mathrm{KCl}, \quad 1.0 \mathrm{MgCl}_{2}, \quad 1.8 \mathrm{CaCl}_{2}$ 
$25 \cdot 0$ glucose, $\mathrm{pH} 7 \cdot 4$. Test solutions were prepared by adding substances in the following concentrations (mM): 0.1 ouabain, 0.02 or $0 \cdot 1$ 2.4-dinitrophenol (DNP), $0.01,0.1$ or $1.0 \mathrm{KCN}, 1.0$ sodium azide, 3.0 $\mathrm{KCl}$. Except for cold, the test solutions were introduced only into the apical side of the chamber. Solutions containing cyanide were bubbled before the cyanide was added and then kept under an atmosphere of $95 \%$ $\mathrm{O}_{2}$ and $5 \% \mathrm{CO}_{2}$. Cold was produced by keeping the reservoirs in an ice bath; by the time the solution reached the chamber, it had warmed to about $15^{\circ} \mathrm{C}$. The temperature in the chamber was continuously monitored with a tele-thermometer probe (YSI, Yellow Springs, $\mathrm{OH}$ ) positioned $1-2 \mathrm{~mm}$ from the tissue. The volume of each side of the chamber was $2.5 \mathrm{ml}$.

\section{Electrodes and Recording}

The placement of electrodes and recording electronics were similar to previously published methods (e.g. Griff and Steinberg, 1982a). In brief, the transepithelial potential (TEP) was measured between the apical and choroidal baths by a pair of agar-Ringer bridges. Conventional microelectrodes were used to measure simultaneously the apical membrane potential, $V_{\mathrm{ap}}$, measured differentially between the microelectrode and the apical bath, and the basal membrane potential, $V_{\mathrm{ba}}$, between the microelectrode and the choroidal bath. The polarity of each membrane potential is inside with respect to outside.

Two resistance ratios were calculated from potential changes induced by injecting $1.0 \mu \mathrm{A}$ current pulses across the tissue. The total resistance across the epithelium, $R_{t}$, was calculated from the currentinduced change in TEP. The ratio of the apical to basal resistances, $a$, was calculated from the ratio of the current-induced changes in apical and basal membrane potential.

All signals were continuously monitored on a storage oscilloscope (model 5111, Tektronix Inc., Beaverton, OR) and a chart recorder (Model 220, Gould Electronics, Cleveland, $\mathrm{OH}$ ). Selected responses were stored digitally at $20 \mathrm{kHz}$ using a VCR recording system (Model IR-2, Indec Systems, Inc., Sunnyvale. CA) and also on computer (Model 158, Zenith Data Systems Corp., St Joseph, MI) using an A/D Board (DASH-16, Metrabyte Corp., Taunton, MA) and a data acquisition program (HSDAS, Quinn-Curtis, Newton Centre. MA). Current-induced potentials used to calculate resistances were digitized on the computer at $100 \mathrm{~Hz}$ : responses to solution changes were digitized at $2 \mathrm{~Hz}$. The digitized responses were plotted on a digital plotter (Model 7550A, Hewlett Packard, San Diego, CA) using a spreadsheet program (Lotus 1-2-3. Lotus Development Corp., Cambridge, MA).

\section{Analysis of Intracellular Recordings}

The resting RPE apical membrane potential of all species examined is larger (more negative inside) than the basal membrane potential, so that the resting TEP is positive when measured outside the apical membrane (subretinal space) with respect to the choroid. The TEP is the difference between the basal and apical membrane potentials: $V_{\mathrm{rad}}-V_{\mathrm{ap}}=\mathrm{TEP}$. Thus, the TEP will decrease if the apical membrane depolarizes or if the basal membrane hyperpolarizes. Analysis of intracellular responses is complicated, however, because the two membranes are electrically connected via a shunt resistance (see Miller and Steinberg, 1977 ; Linsenmeier and Steinberg, 1983). For example, even if a change in potential is generated at only one membrane, the other membrane will polarize in the same direction because current flows through the shunt and across that membrane. The measured change in potential will always be larger at the membrane where the response originates. Thus, if a TEP decrease is caused by an apical depolarization, the apical membrane will depolarize relative to the basal membrane.

By simultaneously monitoring the TEP and both apical and basal membrane potentials. it is relatively easy to determine which membrane's response is larger, even when difference between the apical and basal responses is small. For example, if both membranes hyperpolarize, and the TEP is increasing, the apical hyperpolarization must be larger than the basal hyperpolarization, and a hyperpolarization must therefore originate (be generated) at the apical membrane. On the other hand if both membranes hyperpolarize and the TEP is decreasing, the basal hyperpolarization must be larger than the apical hyperpolarization and a hyperpolarization must originate at the basal membrane. A similar argument can be made for membrane depolarizations. For example, in Figs 1, 2 and 3 , both membranes first depolarized and the TEP decreased. This can occur only if the apical depolarization was greater than the basal depolarization during this time period.

\section{Results}

The results are presented in two sections. The first section demonstrates that metabolic inhibitors increase the basal membrane potential and resistance of the gecko RPE. The second section shows that the inhibitors reduce a basal membrane response that normally can be evoked by changing the potassium concentration in the apical bathing solution, $\left[\mathrm{K}^{+}\right]_{\mathrm{ap}}$.

\section{Effects of Inhibitors on the RPE}

In the initial experiments, the effects of several metabolic inhibitors on the TEP were examined. Potassium cyanide ( $0.1 \mathrm{~mm})$, 2,4-dinitrophenol (DNP, $0.1 \mathrm{~mm})$, and exposure to cold $\left(15^{\circ} \mathrm{C}\right)$ decreased the TEP ; sodium azide ( $1.0 \mathrm{mM})$ increased the TEP. Tissues were exposed to these test solutions for 3-10 min and when the control perfusate was reintroduced, the TEP 


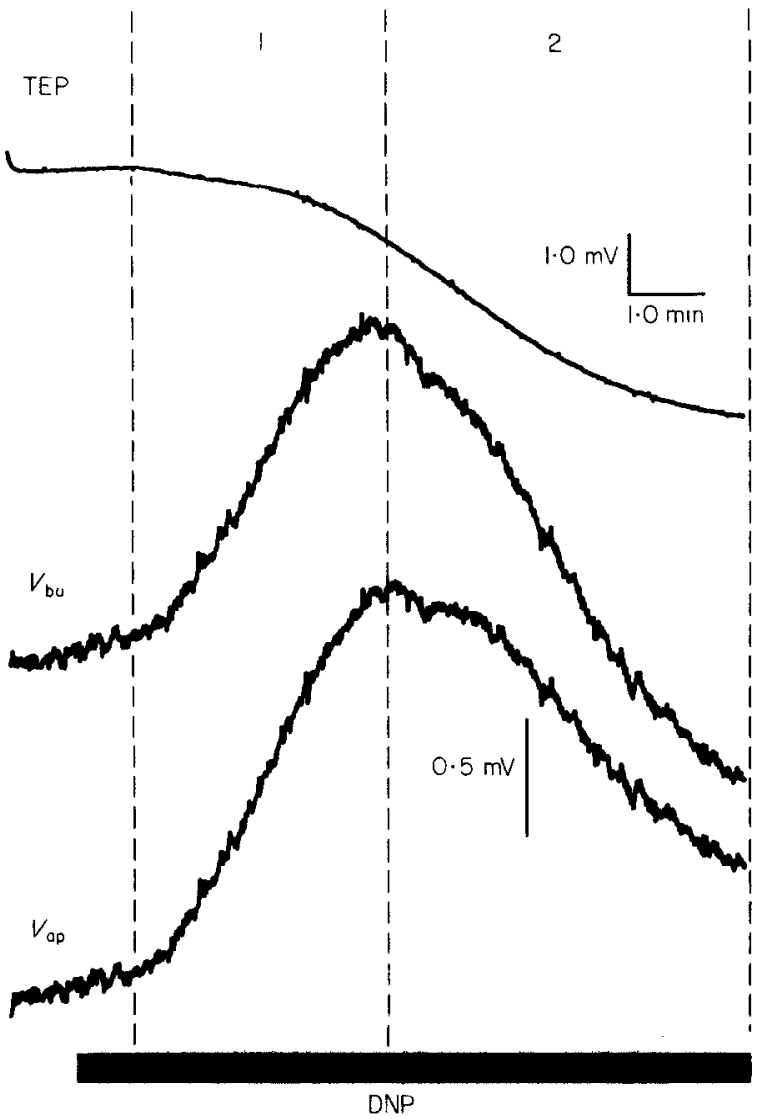

FIG. 1. Trans-epithelial and intracellular responses to DNP. The trans-epithelial potential (TEP) along with the apical and basal membrane potentials $\left(V_{\mathrm{ap}}\right.$ and $\left.V_{\mathrm{ba}}\right)$ were recorded simultaneously while switching the apical perfusate to a test solution containing $0.1 \mathrm{~mm}$ DNP (bar). Initial values of TEP, $V_{\mathrm{ap}}$, and $V_{\mathrm{na}}$ at the start of the record were $17,-75$ and $-58 \mathrm{mV}$, respectively. During period 1, the TEP decreased and both $V_{\mathrm{ap}}$ and $V_{\mathrm{ba}}$ depolarized, with $V_{\mathrm{ap}}$ depolarizing relative to $V_{\mathrm{ba}}$. During period 2 , the TEP continued to decrease but now both membranes hyperpolarized, with $V_{\mathrm{ba}}$ hyperpolarizing relative to $V_{\mathrm{ap}}$. The vertical dashed line dividing period 1 and 2 was drawn at the time both membranes began to hyperpolarize.

recovered almost to its original value (for an example, see Fig. 2). These results were representative of over 50 responses from 23 tissues for DNP, 25 responses from 12 tissues for cyanide, five responses in two tissues for cold, and eight responses from four tissues for azide. Responses could be obtained only when the inhibitors were introduced into the apical or both apical and choroidal perfusates. Adding DNP or cyanide only to the choroidal perfusate for 30-60 min produced no change in TEP even though the inhibitors presumably act intracellularly (see Discussion).

Although the dose/response characteristics for each poison were not examined carefully, a lower concentration of an inhibitor usually produced a smaller, but otherwise similar response. However, for cyanide a low concentration $(1-10 \mu \mathrm{M})$ sometimes increased the TEP (six responses from three tissues), whereas higher concentrations $(0 \cdot 1-1 \cdot 0 \mathrm{mM})$ decreased TEP.

Figure 1 shows the intracellular responses that accompany a TEP decrease evoked by a 10-min
TEP
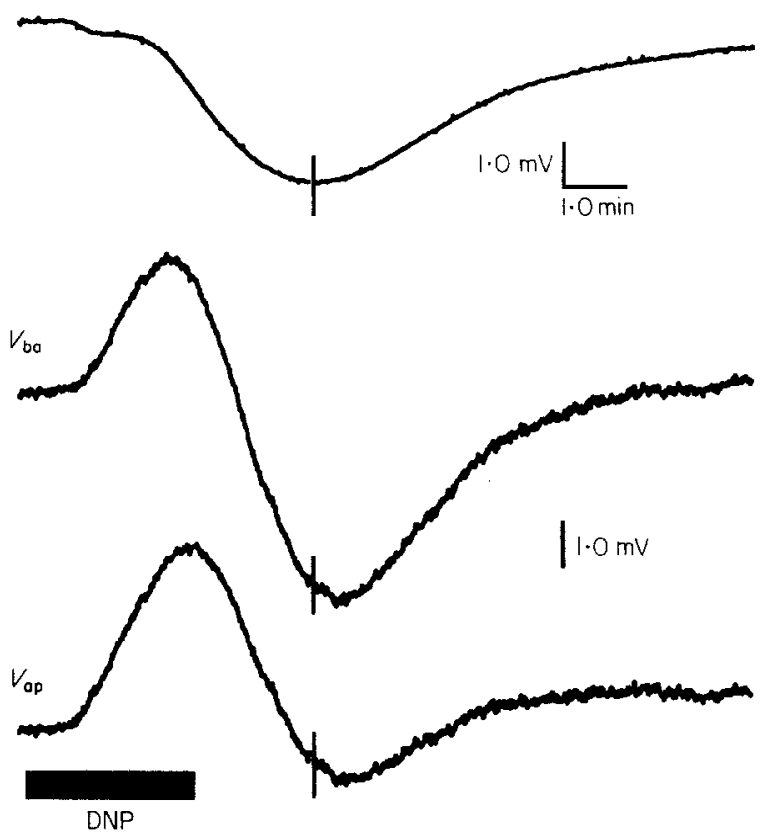

FIG. 2. DNP-evoked response and recovery. TEP, $V_{\text {ap }}$ and $V_{\text {ba }}$ were recorded from the same RPE cell as in Fig. 2 while switching the apical perfusate to one containing $0.1 \mathrm{mM}$ DNP for about $3 \mathrm{~min}$ (bar) and then switching back to the control solution. Adding DNP produced a similar sequence of potential changes as shown in Fig. 1. After the control solution was reintroduced, the TEP began to increase (vertical lines through responses). Initially, the TEP increased because $V_{\mathrm{ap}}$ hyperpolarized relative to $V_{\mathrm{ba}}$. During most of the TEP recovery, however, $V_{\mathrm{ba}}$ depolarized relative to $V_{\mathrm{ap}}$. All potentials returned approximately to the control values given in Fig. 1 .

exposure to DNP in the apical perfusate. The TEP decrease could be separated into two events. During the initial TEP decrease (period 1), the apical membrane depolarized relative to the basal membrane. This indicates that the TEP decrease during period 1 was generated by a depolarization of the apical membrane, and that the basal membrane depolarized passively because current was shunted across its resistance (see Materials and Methods).

The beginning of period 2 was set as the time when the RPE membranes stopped depolarizing and started hyperpolarizing. During period 2 , in the continued presence of DNP, the TEP continued to decrease, but now the basal membrane hyperpolarized relative to the apical membrane. This indicates that the TEP decrease during period 2 was generated by a hyperpolarization of the basal membrane and that the apical membrane hyperpolarized passively because current was shunted across its resistance. Thus, the TEP decrease evoked by DNP was produced first by a depolarization generated at the apical membrane followed by a hyperpolarization generated at the basal membrane. This pattern of response was observed consistently (eight responses from five cells in four tissues).

Figure 2 shows the response to a brief $(3 \mathrm{~min}$ ) exposure to DNP to demonstrate that the effects of 


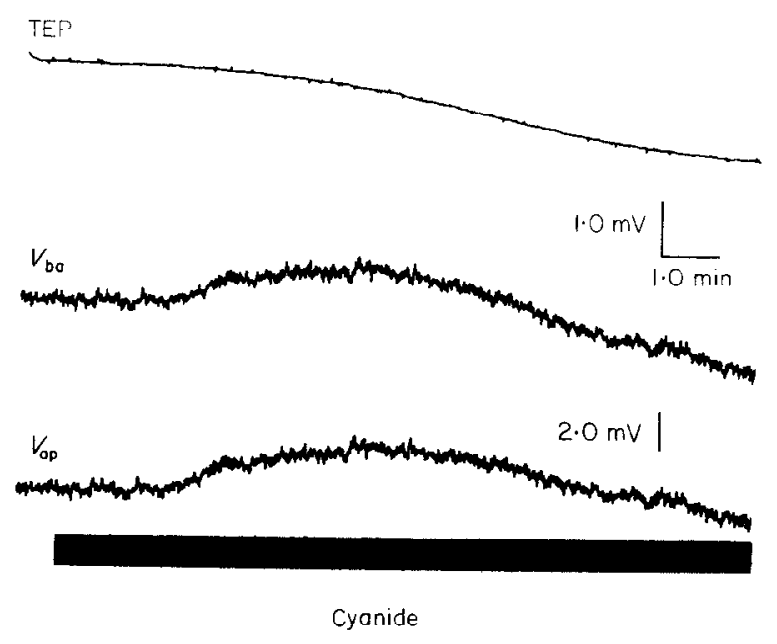

FIG. 3. Responses to cyanide. TEP, $V_{\mathrm{ap}}$ and $V_{\mathrm{ba}}$ were recorded as in Fig. 1 (initial values: $11,-83$ and $-72 \mathrm{mV}$, respectively) while the apical perfusate only was switched to a test solution containing $0 \cdot 1 \mathrm{~mm}$ potassium cyanide (bar) for the duration of the recording. During the initial TEP decrease $V_{\mathrm{ap}}$ depolarized relative to $V_{\mathrm{ba}}$; during the subsequent TEP decrease $V_{\mathrm{ba}}$ hyperpolarized relative to $V_{\mathrm{ap}}$.

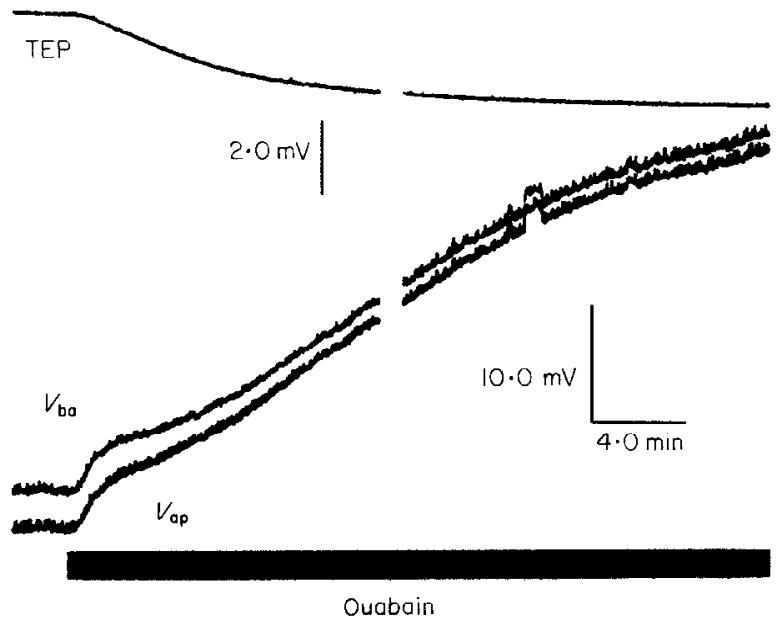

FIG. 4. Responses to ouabain. TEP, $V_{\mathrm{ap}}$ and $V_{\mathrm{ba}}$ (initial values: $6,-80$ and $-74 \mathrm{mV}$, respectively) were recorded as in Fig. 1 while the apical perfusate only was switched to a test solution containing $0.3 \mathrm{~mm}$ ouabain (bar). Ouabain decreased the TEP during which $V_{\mathrm{ap}}$ depolarized relative to $V_{\mathrm{m}}$; a relatively rapid depolarization was followed by a slower, maintained depolarization. The response was digitized in two parts causing a $1 \mathrm{~min}$ break in the record shown.

DNP are reversible. DNP decreased the TEP, generated initially by a depolarization originating at the apical membrane, followed by a hyperpolarization originating at the basal membrane. When the solution was switched back to the control Ringer's, the TEP began to increase. The vertical lines through the recordings in Fig. 2 indicate the time at which the TEP started to increase. Initially, this TEP increase was generated by a hyperpolarization of the apical membrane relative to the basal. During most of the TEP increase, however, the basal membrane depolarized relative to the apical. This indicates that a depolarization originating at the basal membrane generated most of the TEP recovery.
Similar intracellular response patterns during recovery were observed in four responses in two cells from different tissues.

The next experiments demonstrate that the same response pattern that was evoked by DNP could also be produced by potassium cyanide (Fig. 3). When $0 \cdot 1$ mu cyanide was added to only the apical perfusate. the TEP decreased. During the initial TEP decrease, the apical membrane depolarized relative to the basal membrane; during the subsequent TEP decrease the basal membrane hyperpolarized relative to the apical. Similar results were obtained for ten responses from five cells in two tissues. Thus, cyanide also caused a TEP decrease that can be divided into two events. The initial TEP decrease was generated by a depolarization of the apical membrane; the subsequent TEP decrease was generated by a hyperpolarization of the basal membrane.

Cold produced a pattern of apical and basal membrane potential changes similar to those shown above for DNP and cyanide but the onset of the response was slower. Intracellular recordings were obtained for four responses from one cell. Thus, DNP, cyanide, and cold all produced a TEP decrease generated in part from a depolarization that originated at the apical membrane and in part from a hyperpolarization that originated at the basal membrane.

The initial apical depolarization caused by DNP, cyanide, and cold is the polarity one would expect if these metabolic inhibitors slowed the electrogenic, apical $\mathrm{Na}^{+}-\mathrm{K}^{+}$pump (Miller, Steinberg and Oakley, 1978). To test whether inhibition of the $\mathrm{Na}^{-}-\mathrm{K}^{\prime}$ pump itself produced the subsequent basal hyperpolarization, responses to ouabain were investigated. Figure 4 shows responses evoked by switching the apical perfusate to a test solution containing $0 \cdot 1 \mathrm{~mm}$ ouabain. After adding ouabain, the apical membrane depolarized relative to the basal membrane, indicating that the response was generated by the apical membrame and passively shunted to the basal membrane. No basal membrane hyperpolarization was observed with ouabain (four cells in four tissues). Thus. inhibition of the $\mathrm{Na}^{+}-\mathrm{K}^{+}$pump with ouabain was not sufficient to generate a basal hyperpolarization.

If the initial apical depolarization evoked by DNP. cyanide, and cold does reflect inhibition of the $\mathrm{Na}^{+}-\mathrm{K}^{+}$ pump, pretreatment with ouabain should eliminate this initial response, and hopefully isolate the TEP decrease generated by the basal hyperpolarization. However, after pretreating with ouabain, or in the continued presence of ouabain, the entire DNP-evoked TEP response was abolished or greatly reduced ( $n=$ 9). Intracellular recordings were obtained in three tissues to examine the effects of DNP on the apical and basal membrane potentials in the presence of ouabain. No DNP-evoked apical depolarization could be detected on the slowly depolarizing baseline caused by the ouabain. In one experiment, a small (less than $1 \mathrm{mV}$ ) 

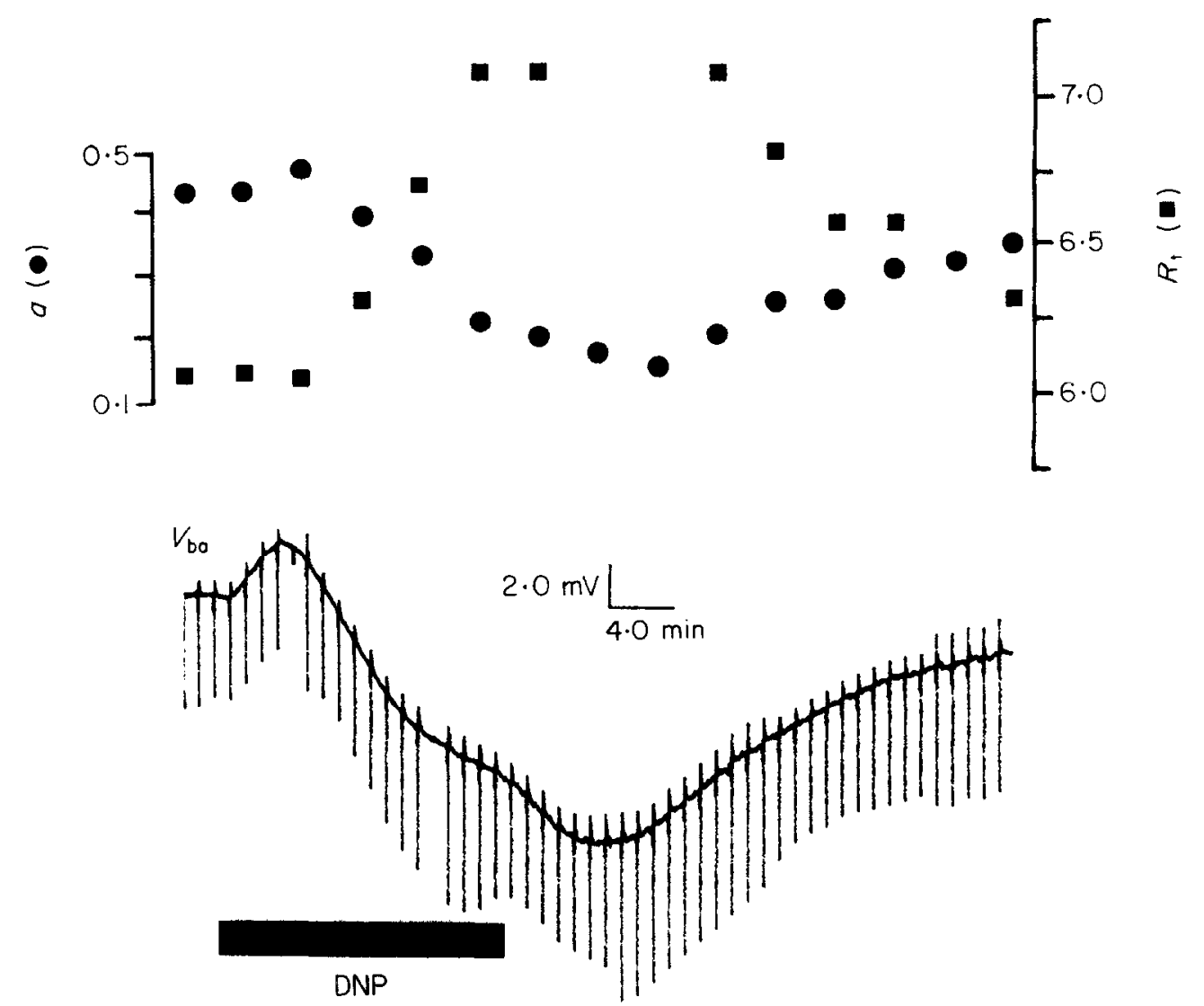

FIG. 5. Changes in RPE resistance in DNP. TEP, $V_{\mathrm{ap}}$ and $V_{\mathrm{ba}}$ were recorded as in Fig. 2 (initial values: $14,-77$ and $-63 \mathrm{mV}$, respectively) while the apical perfusate only was switched to a test solution containing $0.1 \mathrm{~mm}$ DNP (bar): to simplify the figure, only $V_{\mathrm{ba}}$ is shown. $1 \mu \mathrm{A}$ pulses of current were injected across the tissue every minute to measure current-induced potentials (iR drops. negative transients in $\left.V_{\mathrm{ba}}\right) . R_{\mathrm{t}}(\mathrm{k} \Omega)$ was calculated from the $i R$ drops across the tissue, and $a$ is the ratio of the $i R$ drops across the apical and basal cell membranes. $R_{t}$ (filled squares) and a (filled circles) are plotted as a function of time. During the DNP-evoked basal hyperpolarization, $R_{t}$ increased and $a$ decreased. Both $R_{t}$ and $a$ recovered after DNP was washed out.

basal hyperpolarization generated a small DNP-evoked TEP decrease. In an additional experiment, pretreating the RPE with DNP, which decreased TEP from 13 to $4 \mathrm{mV}$, blocked any further response to ouabain. These results support the hypothesis that the initial apical depolarization evoked by DNP was caused by inhibition of the apical $\mathrm{Na}^{+}-\mathrm{K}^{+}$pump. The mechanism by which inhibition of the $\mathrm{Na}^{+}-\mathrm{K}^{+}$pump with ouabain also prevented the DNP-evoked basal hyperpolarization is not known.

To investigate further the responses evoked by metabolic inhibitors, current was injected across the tissue and resistance ratios were calculated from measurements of the current-induced potentials (see Materials and Methods). Figure 5 shows the changes in the trans-epithelial resistance, $R_{\mathrm{t}}$ (filled squares), and the ratio of apical to basal resistance, $a$ (filled circles) that occur during exposure to DNP (bar). The basal membrane potential (bottom trace) that was recorded simultaneously is shown for reference. The TEP and apical membrane potential were also recorded but are not shown in the figure.

Addition of DNP caused an increase in $R_{t}$ and a decrease in the ratio $a$. After DNP was removed, both RPE resistance ratios returned to their control values.
Similar results were found in four responses from two cells in two tissues. An increase in $R_{\mathrm{t}}$ by DNP was measured extracellularly in 12 other tissues. The resistance ratios began to change at approximately the time when the basal membrane started to hyperpolarize. After switching back to the control solution, the resistance ratios began to recover when the basal membrane started to depolarize. Thus, the resistance changes followed approximately the same time course as the voltage changes generated at the basal membrane.

An increase in $R_{t}$ indicates that at least one of the component resistances must increase, and the decrease in the ratio $a$ requires that either the apical resistance decrease, or the basal resistance increase, or both. The simplest explanation to account for the changes in both $R_{t}$ and $a$ is that DNP produced an increase of the basal resistance.

Cyanide produced similar resistance changes. For the intracellular responses shown in Fig. $3, R_{\mathrm{t}}$ increased from $2 \cdot 3$ to $2 \cdot 5 \mathrm{k} \Omega$ and the ratio $a$ decreased from 0.39 to 0.35 . Increases in $R_{\mathrm{t}}$ were found in seven other tissues with cyanide; the largest increase was from 6.75 to $10.0 \mathrm{k} \Omega$. The decrease in $a$ was observed in one other cell. 
Sodium azide produced a different response from the other metabolic inhibitors; it increased TEP. Figure 6 shows that this TEP increase was generated by a depolarization generated at the basal membrane. During this response $R_{\mathrm{t}}$ decreased from 6.2 to $5.4 \mathrm{k} \Omega$ and the ratio $a$ increased from 0.44 to 0.61 . Similar

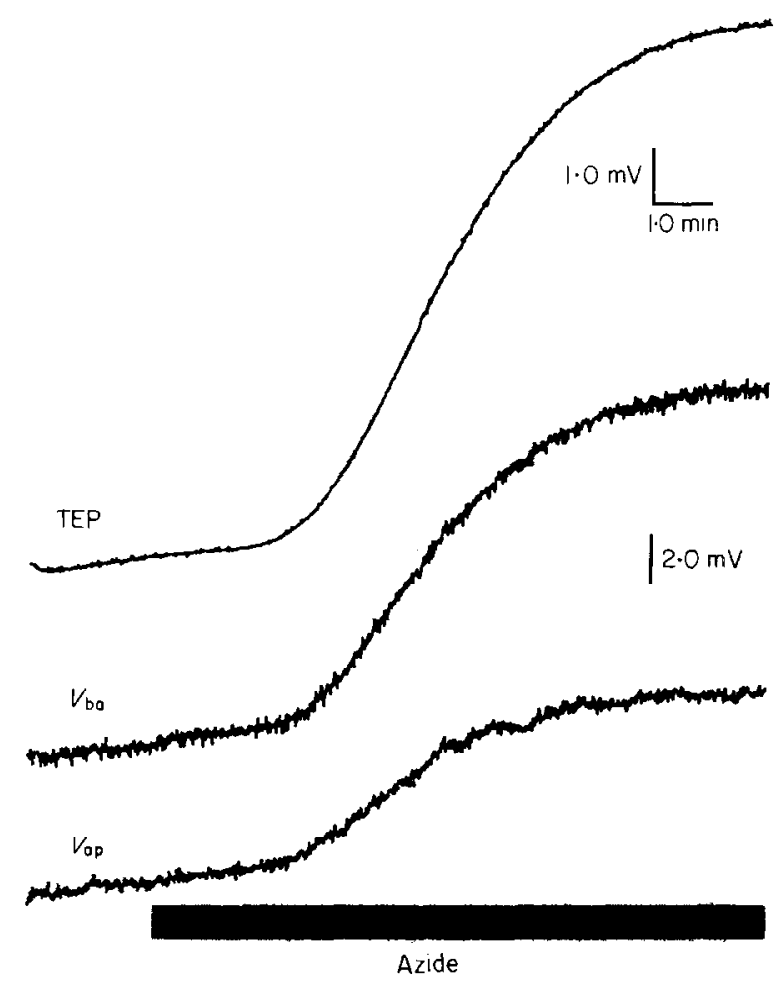

Fig. 6. Responses to azide. TEP, $V_{\mathrm{ap}}$ and $V_{\mathrm{ba}}$ were recorded as in Fig. 1 (initial values: $19,-71$ and -52 respectively) while the apical perfusate only was switched to a test solution containing $1.0 \mathrm{~mm}$ azide (bar). Azide increased the TEP because $V_{\mathrm{ba}}$ depolarized relative to $V_{\mathrm{ap}}$. results were obtained in three responses from one cell in another tissue. These results with azide confirm results in the intact cat preparation (Linsenmeier and Steinberg, 1987) and in vitro preparation of chick retina (Gallemore and Steinberg, 1989).

\section{The Effects of Inhibitors on $\left[\mathrm{K}^{+}\right]_{a_{p}}$-evoked Basal Membrane Potentials}

Because metabolic inhibitors altered the potential and resistance of the basal membrane, the effects of these inhibitors on another basal membrane response were examined. When $\left[\mathrm{K}^{+}\right]_{a p}$ is increased from 2 to $5 \mathrm{~mm}$, a TEP decrease generated by an apical depolarization is followed by a delayed increase in TEP generated by a basal depolarization termed the delayed basal depolarization (Griff and Steinberg, 1984). Upon returning $\left[\mathrm{K}^{+}\right]_{\mathrm{ap}}$ from 5 to $2 \mathrm{mM}$, a TEP increase generated by an apical hyperpolarization is followed by a TEP decrease generated by a delayed basal hyperpolarization. The delayed basal hyperpolarization was accompanied by a decrease in $R_{\mathrm{t}}$ and an increase in the ratio $a$. Similar delayed basal membrane changes have also been demonstrated in preparations of neural retina-RPE-choroid in which light causes the $\left[\mathrm{K}^{+}\right]_{0}$ decrease in the subretinal space (Griff and Steinberg, 1984; Linsenmeier and Steinberg, 1984; Joseph and Miller, pers. commun). The delayed basal hyperpolarization generated by light contributes to the fast oscillation of the DC-ERG (Steinberg et al., 1985).

Figure 7 shows TEP responses evoked by increasing $\left[\mathrm{K}^{+}\right]_{a \mathrm{p}}$ from 2 to $5 \mathrm{mM}$ (bars) in control (top row), in test conditions (middle row), and back in control (bottom row, recovery) for three test conditions. The
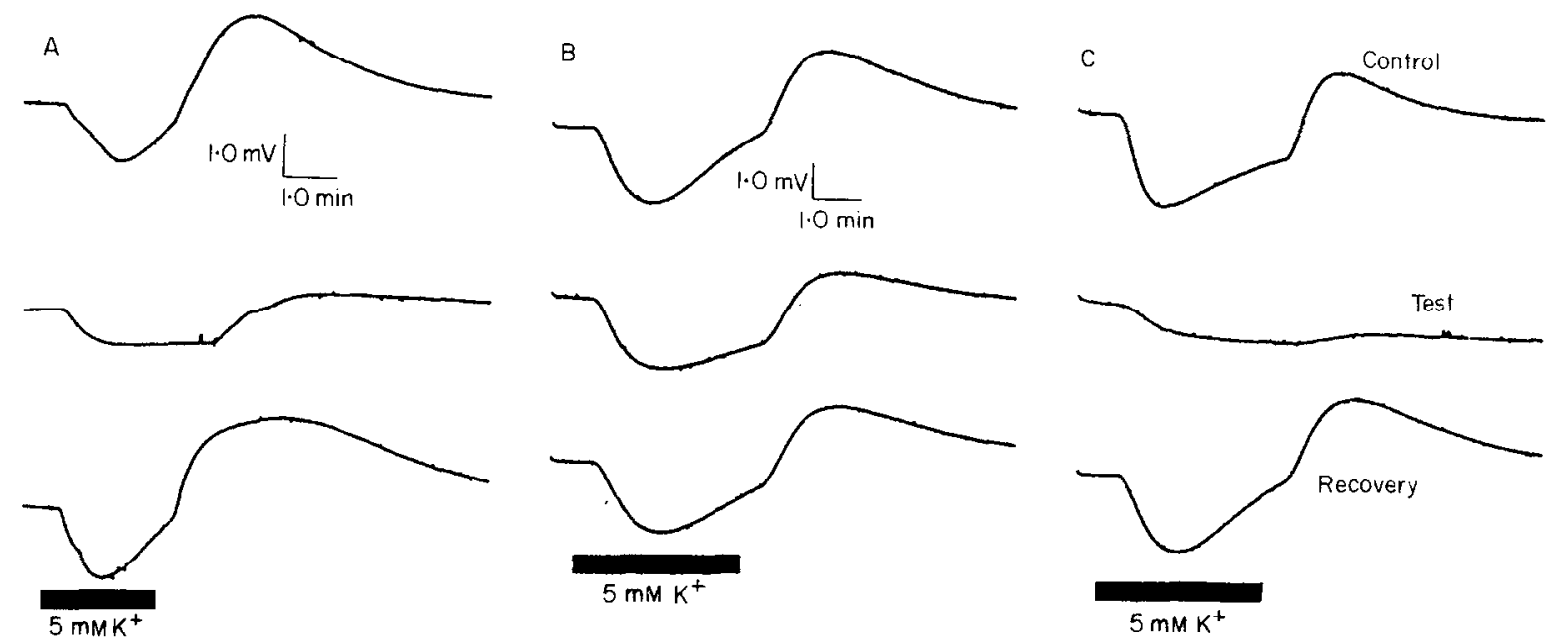

Fig. 7. The effect of cyanide and DNP on the $\left[\mathrm{K}^{+}\right]_{\mathrm{ap}}$-evoked response. The TEP was recorded while the apical perfusate was switched from $2 \mathrm{mM}$ to $5 \mathrm{~mm}\left[\mathrm{~K}^{+}\right]_{\mathrm{ap}}$ and back (bars) in each set of recordings. The top trace in each column is a control $\left[\mathrm{K}^{+}\right]_{\mathrm{aI}}{ }^{-}$ evoked response (initial values in $A, B$ and $C$ were $6.0,3 \cdot 0$ and $5.5 \mathrm{mV}$, respectively). In the control responses, elevating $\left[\mathrm{K}^{+}\right]_{\mathrm{ai}}$ caused a TEP decrease followed by a delayed TEP increase; switching back to $2 \mathrm{~mm}\left[\mathrm{~K}^{+}\right]_{\mathrm{ap}}$ produced an increase followed by a delayed decrease (overshool) of the TEP. After recording each control response, the solution was switched to a test solution, and the middle traces show $\left[\mathrm{K}^{+}\right]_{\mathrm{ap}}$-evoked responses in the continued presence of the test substance $(\mathrm{A}, 0 \cdot 1 \mathrm{~mm}$ potassium cyanide: B. $20 \mu \mathrm{M}$ DNP: C. $0 \cdot 1 \mathrm{~mm}$ DNP). In $0.1 \mathrm{~mm}$ cyanide (A) and $0 \cdot 1 \mathrm{~mm}$ DNP (C), elevating $\left[\mathrm{K}^{+}\right]_{\mathrm{ap}}$ caused only a monotonic TEP decrease; the delayed TEP increase and overshoot were eliminated. Twenty $\mu \mathrm{M}$ DNP (B) reduced the delayed TEP increase and overshoot. Bottom traces show the recovery of the control response after switching back to control Ringer's. 

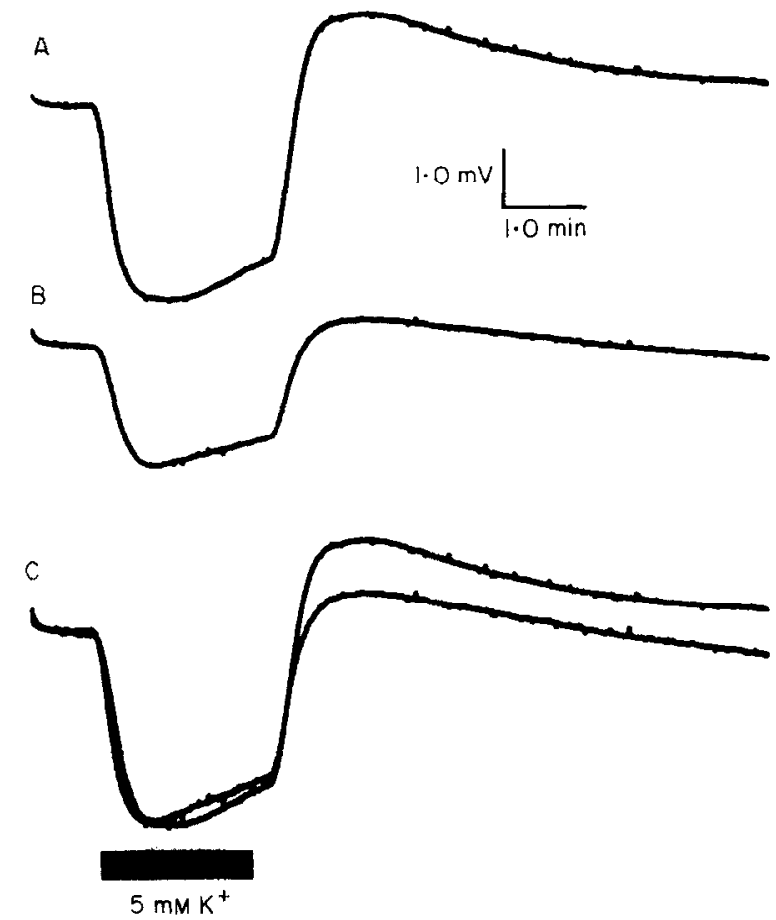

FIG. 8. Effects of ouabain on the $\left[\mathrm{K}^{+}\right]_{\mathrm{ap}}$-evoked response. The TEP was recorded as in Fig. 7 while the apical perfusate was switched from 2 to $5 \mathrm{mM}\left[\mathrm{K}^{+}\right]_{\mathrm{ap}}$ and back to $2 \mathrm{mM}$ as indicated by the bar. Trace $A$ shows the control response. Prior to the response shown in $\mathrm{B}, 0.1 \mathrm{~mm}$ ouabain was added to the apical solution: this caused a TEP decrease from 18 to $5.5 \mathrm{mV}$. Trace $\mathrm{B}$ shows the $\left[\mathrm{K}^{+}\right]_{\mathrm{gp}}$-evoked response recorded in the continued presence of ouabain. To compare the waveforms of these responses, the response recorded in ouabain was scaled to the same peak amplitude as the control and the two superimposed (C).

control response, described in the previous paragraph, consisted of a TEP decrease and delayed increase (return toward the baseline) when $\left[\mathrm{K}^{+}\right]_{\mathrm{av}}$ was increased from 2 to $5 \mathrm{mM}$, and a TEP increase and delayed decrease (overshoot) when $\left[\mathrm{K}^{+}\right]_{\mathrm{av}}$ was returned to $2 \mathrm{~mm}$. In the presence of $0.1 \mathrm{~mm}$ cyanide (column $\mathrm{A}$, test), increasing $\left[\mathrm{K}^{+}\right]_{\mathrm{ap}}$ caused only a TEP decrease; the delayed TEP increase normally generated by a delayed basal depolarization was abolished. Returning from 5 to $2 \mathrm{mM}$ produced a TEP increase with a very small overshoot. After removing cyanide from the solution. increasing $\left[\mathrm{K}^{+}\right]_{a p}$ again evoked a control response (column A, recovery). In one tissue $\left[\mathrm{K}^{+}\right]_{\mathrm{sp}}$-evoked changes in $R_{\mathrm{t}}$ were also measured. In the control response, $R_{t}$ decreased $15 \%$ during the delayed TEP increase; in the presence of $0.1 \mathrm{~mm}$ cyanide, the delayed TEP increase was partly blocked and $R_{\mathrm{t}}$ decreased by only $2 \%$.

Figure 7 also shows that $0.1 \mathrm{~mm}$ DNP (column B, test) reversibly abolished the delayed TEP increase normally evoked by increasing $\left[\mathrm{K}^{+}\right]_{\mathrm{ap}}$. A lower dose of DNP ( $20 \mu \mathrm{M}$ DNP, column $C$, test) reduced the delayed TEP increase and the overshoot. Both azide (1.0 mM) and cold $\left(15-18^{\circ} \mathrm{C}\right)$ also decreased or abolished the delayed TEP responses (not shown). Thus, all the metabolic inhibitors used in this study either reduced or abolished the $\left[\mathrm{K}^{+}\right]_{\mathrm{ap}}$-evoked delayed TEP responses.

The effects of each metabolic inhibitor used on the $\left[\mathrm{K}^{+}\right]_{\mathrm{ap}}$-evoked, delayed TEP responses could have been mediated by an inhibition of the apical $\mathrm{Na}^{+}-\mathrm{K}^{+}$pump. To determine the effect of pump inhibition on the $\left[\mathrm{K}^{+}\right]_{\mathrm{ap}}$-evoked TEP responses, the tissue was pretreated with $0.1 \mathrm{~mm}$ ouabain. $\left[\mathrm{K}^{+}\right]_{\mathrm{ap}}$ was then changed from 2 to $5 \mathrm{mM}$ and back to $2 \mathrm{mM}$ in the continued presence of ouabain in the apical perfusate. As shown in Fig. 8. ouabain did not abolish the delayed TEP increase although both the initial TEP decrease and the delayed TEP increase toward baseline were reduced. When the $\left[\mathrm{K}^{+}\right]_{\mathrm{ap}}$-evoked responses before and during ouabain exposure were scaled to the same peak amplitude and superimposed (bottom traces), the waveforms of the responses were similar.

\section{Discussion}

\section{Effects of Metabolic Inhibitors}

DNP, cyanide and cold each decreased the transepithelial potential (TEP) of the gecko RPE. Miller et al. (1978) found a similar TEP decrease in the bullfrog RPE, and intracellular recordings showed that it was generated by a depolarization of the RPE apical membrane. The results in this paper show that the responses of the gecko RPE are more complex since the TEP decrease was accompanied by membrane potential changes that originated from both the apical and basal membranes (also see Steinberg and Miller. 1979).

In the gecko, DNP, cyanide and cold each produced a depolarization of apical membrane followed by a hyperpolarization of the basal membrane (Figs 1. 2 and 3). The gecko, but not the bullfrog, also exhibits a $\left[\mathrm{K}^{+}\right]_{\mathrm{ap}}$-evoked basal membrane response (Griff and Steinberg, 1984) and a light peak basal depolarization (Kikawada, 1968; Griff and Steinberg, 1982a). Thus, the DNP-evoked basal hyperpolarization may reflect a property of the 'mammalian type' RPE.

DNP, cyanide and cold hyperpolarized the basal membrane. This indicates that the basal membrane normally must be kept depolarized by mechanisms and/or cell functions that are sensitive to these inhibitors. This basal depolarization will contribute to the resting trans-epithelial potential, and in turn, to the corneal-positive standing potential.

The basal hyperpolarization evoked by DNP, cyanide, and cold was accompanied by an increase in the total resistance, $R_{\mathrm{t}}$, and a decrease in the ratio of apical to basal membrane resistance, $a$. These resistance changes are most easily explained by an increase in basal membrane resistance (decrease in conductance). The significance of this conductance change to the basal membrane potential change is unclear, however, since the passive electrical properties of the RPE basal membrane of gecko are unknown. 
The conductance decrease could cause the hyperpolarization or could result from the hyperpolarization as a voltage-sensitive conductance.

The apical depolarization that caused the initial TEP decrease with DNP, cyanide or cold probably was due to inhibition of the electrogenic apical $\mathrm{Na}^{+}-\mathrm{K}^{+}$pump. However, inhibition of the pump with ouabain (Fig. 4) did not produce a basal hyperpolarization. Rather, as has been demonstrated in several other species (e.g. Miller et al., 1978; Joseph and Miller, pers. commun.). there was a rapid apical depolarization that reflects elimination of the electrogenic pump's contribution to the apical membrane potential, followed by a slower depolarization as the concentration gradients for $\mathrm{Na}^{+}$ and $\mathrm{K}^{+}$presumably decrease.

The role of the $\mathrm{Na}^{+}-\mathrm{K}^{+}$pump was further investigated by examining interactions between DNP and ouabain. Pretreatment of the tissue with ouabain abolished subsequent responses to DNP. While this effect of ouabain supports the hypothesis that DNP inhibited the $\mathrm{Na}^{+}-\mathrm{K}^{+}$pump, it also suggests that a functional pump is required for the subsequent basal hyperpolarization, possibly for maintaining intracellular ion concentrations. It was not possible to analyze the membrane changes evoked by DNP within the first 10 min after adding ouabain, before ion concentrations change appreciably, because both membranes were depolarizing rapidly.

The mechanism(s) by which metabolic inhibitors produce a change in the RPE basal membrane are unknown at present. The fact that DNP, cyanide and cold produced the same pattern of RPE membrane responses may suggest a common mechanism for these inhibitors. One possibility is a direct effect on the basal membrane. However, it is unlikely that a ring compound such as DNP, an ion such as cyanide and a general perturbation such as cold would all produce the same direct effect. Furthermore, the observation that the inhibitors were effective in altering the basal membrane potential when applied to the apical perfusate suggests an intracellular step is involved. Inhibition of ATP production in the mitochondria is a plausible mechanism (Slater, 1967).

The effects of sodium azide on the gecko RPE were different from the other inhibitors. Instead of a TEP decrease generated in part by a basal hyperpolarization, azide depolarized the basal membrane so that the TEP increased. Azide decreased the apparent basal membrane resistance whereas the other inhibitors increased apparent basal resistance. The azideevoked responses in this study were similar to responses evoked in cat by injection of azide (Linsenmeier and Steinberg, 1987) and responses in chick evoked by adding azide only to the choroidal perfusate (Gallemore and Steinberg, 1989b). Azide may act by mechanisms unrelated to its role as a metabolic inhibitor.

The present data do not allow one to distinguish whether the change in basal membrane potential evoked by each inhibitor was a direct consequence of metabolic poisoning or an indirect consequence of intracellular ion concentration or $\mathrm{pH}$ changes. The activity of an electrogenic pump on either membrane could be reduced or a conductance or electrogenic cotransport mechanism could be affected. The basal membrane responses could also reflect concentration changes in the unstirred layer in the subchoroidal space caused by changes in ion transport across either the apical or basal membrane (Immel and Steinberg, 1986).

The gecko RPE did not respond when inhibitors were added only to the choroidal perfusate, probably because the inhibitors did not reach the basal membrane through the choroid. In previous experiments (Griff and Steinberg, unpubl. obs.), a tenfold increase in $\mathrm{K}^{+}(n=3)$, a tenfold decrease in $\mathrm{HCO}_{3}^{-}$ $(n=4)$ and a tenfold decrease in $\mathrm{Cl}^{-}(n=2)$ had little effect on TEP when only the choroidal solution was switched. Light microscopy of the gecko choroid revealed continuous layers of darkly stained cells that may block free diffusion (Griff and Steinberg, unpubl. obs.). Addition of several types of collagenases, elastase, or hyaluronidase to the basal perfusate did little to remove the diffusion barrier. Thus, further investigation of the gecko basal membrane will be difficult.

\section{The Delayed Basal Response}

The mechanisms by which $\left[\mathrm{K}^{+}\right]_{\mathrm{qp}}$ produces a delayed basal response are still unclear. Preliminary evidence suggested that a change in intracellular potassium and/or chloride was required (Griff and Steinberg. 1982b), and a change in apparent basal conductance has been shown (Griff and Steinberg, 1984). In bovine RPE, Joseph and Miller (pers. commun.) proposed that a decreased influx of chloride across the apical membrane via a $\mathrm{Na}, \mathrm{K}, \mathrm{Cl}$ cotransporter led to a decrease in intracellular chloride that hyperpolarized the basal membrane. The present study (Fig. 7) shows that all the metabolic inhibitors used reversibly abolished the $\left[\mathrm{K}^{+}\right]_{\mathrm{ap}}$-evoked delayed TEP responses whereas ouabain did not (Fig. 8).

DNP and cyanide each hyperpolarized the basal membrane and increased its apparent resistance. However, it is not yet known how these effects relate to the $\left[\mathrm{K}^{+}\right]_{\mathrm{av}}$-evoked basal responses. Azide, on the other hand, depolarized the basal membrane and decreased its apparent resistance. Azide also blocks the light peak (Linsenmeier and Steinberg, 1987).

A recent report shows that the anion transport blocker DIDS hyperpolarized the chick RPE basal membrane and increased apparent basal membrane resistance, suggesting a block of chloride conductance (Gallemore and Steinberg, 1989a). DIDS also abolished the slow basal depolarization that generates the light peak (Gallemore and Steinberg, 1989b). It is not 
known whether DIDS affects the same basal membrane mechanism as DNP or cyanide.

\section{Acknowledgments}

We thank Rob Linsenmeier, Roy Steinberg, Burks Oakley, II. Bret Hughes and Ron Gallemore for their helpful comments on an earlier draft of this manuscript and Laura McKibben for technical assistance. This research was supported by the National Eye Institute (EY05893) and the University of Cincinnati Research Council.

\section{References}

Gallemore, R. P, and Steinberg, R. H. (1989a). Effects of DIDS on the chick retinal pigment epithelium. I. Membrane potentials. apparent resistances and mechanisms. J. Neurosci. 9, 1968-76.

Gallemore, R. P. and Steinberg, R. H. (1986b). Effects of DIDS on the chick retinal pigment epithelium. II. Mechanism of the light peak and other responses originating at the basal membrane. J. Neurosci. 9. 1977-84

Griff, E. R. and Steinberg, R. H. (1982a). Origin of the light peak: in vitro study of Gekko gekko. J. Physiol. 33, 637-52.

Griff, E. R. and Steinberg, R. H. (1982b). RPE basal membrane hyperpolarization follows light-evoked, $\mathrm{K}^{+}$-dependent, apical membrane hyperpolarization. Soc. Neurosci. abstr. 8, 44.

Griff, E. R. and Steinberg, K. H. (1984). Changes in apical $\left[\mathrm{K}^{+}\right]$produce delayed basal membrane responses of the retinal pigment epithelium in the gecko. J. Gen. Physiol. 83, 193-211.

Immel, J. and Steinberg, R. H. (1986). Spatial buffering of $\mathrm{K}^{+}$ by the retinal pigment epithelium in frog. J. Neurosci. 56, 3197-204.

Kikawada, N. (1968). Variations in the corneo-retinal standing potential of the vertebrate eye during light and dark adaptations. Jpn. J. Physiol. 18, 687-702.
Linsenmeier. R. A. and Steinberg, R. H. (1983). A lightevoked interaction of apical and basal membranes of the retinal pigment epithelium: c-wave and light peak. J. Neurophysiol. 50, 136-47.

Linsenmeier, R. A. and Steinberg, R. H. (1984). Delayed basal hyperpolarization of cat retinal pigment epithelium and its relation to the fast oscillation of the DC electroretinogram. J. Gen. Physiol. 83, 213-32.

Linsenmeier, K. A. and Steinberg, K. H. (1987). Mechanisms of azide induced increases in the c-wave and standing potential of the intact cat eye. Vision Res. 27, 1-8.

Marmor, M. F. and Lurie, M. (1979). Light induced response of the retinal pigment epithelium. In The Retinal Pigment Epithelium (Eds Zinn, K. M. and Marmor. M. F.). Pp. 226-44. Harvard University Press: Cambridge, MA.

Miller, S. S. and Steinberg, R.H. (1977). Passive ionic properties of frog retinal pigment epithelium. J. Membr. Biol. 36. 337-72.

Miller, S. S., Steinberg, R. H. and Oakley. B. II. (1978). The electrogenic sodium pump of frog retinal pigment epithelium. J. Membr. Biol. 44, 259-79.

Oakley. B. II. (1977). Potassium and the photoreceptordependent pigment epithelial hyperpolarization. J. Gen. Physiol. 70, 405-25.

Slater, E. C. (1967). Application of inhibitors and uncouplers for a study of oxidative phosphorylation. Methods Enzymol. 10, 48-57.

Steinbery, R. H., Linsenmeier, R. A. and Griff, E. R. (1985). Retinal pigment epithelial cell contributions to the electroretinogram and electrooculogram. In Progress in Retinal Research (Eds Osborne, N. N. and Chader, G. J.). Pp. 33-66. Pergamon Press: New York.

Steinberg, K. H. and Miller, S. S. (1979). Transport and membrane properties of retinal pigment epithelium. In The Retinal Pigment Epithelium (Eds Zinn. K. M. and Marmor. M. F.). Pp. 205-25.

Van Norren. D. and Heynen, H. (1986). Origin of the fast oscillation in the electroretinogram of the Macaque. Vision Res. 26, 569-75. 